\title{
DEVELOPMENT OF A COST EFFECTIVE MINI AUTONOMOUS UNDERWATER VEHICLE
}

Chiu-Feng Lin

Professor, Department of Vehicle Engineering, National Pingtung University of Science and Technology, 1, Hseuh Fu Road, Neipu, Pingtung, Taiwan., chiufeng@mail.npust.edu.tw

Chyuan-Yow Tseng

Professor, Department of Vehicle Engineering, National Pingtung University of Science and Technology, 1, Hseuh Fu Road, Neipu, Pingtung, Taiwan.

Follow this and additional works at: https://jmstt.ntou.edu.tw/journal

Part of the Electrical and Computer Engineering Commons

\section{Recommended Citation}

Lin, Chiu-Feng and Tseng, Chyuan-Yow (2006) "DEVELOPMENT OF A COST EFFECTIVE MINI AUTONOMOUS UNDERWATER VEHICLE," Journal of Marine Science and Technology. Vol. 14: Iss. 2, Article 7.

DOI: $10.51400 / 2709-6998.2065$

Available at: https://jmstt.ntou.edu.tw/journal/vol14/iss2/7

This Research Article is brought to you for free and open access by Journal of Marine Science and Technology. It has been accepted for inclusion in Journal of Marine Science and Technology by an authorized editor of Journal of Marine Science and Technology. 


\title{
Short Paper
}

\section{DEVELOPMENT OF A COST EFFECTIVE MINI AUTONOMOUS UNDERWATER VEHICLE}

\author{
Chiu-Feng Lin* and Chyuan-Yow Tseng*
}

Key words: autonomous underwater vehicle, obstacle avoidance, image processing.

\begin{abstract}
This paper describes the development of a cost effective mini autonomous underwater vehicle. The mini size of the vehicle is achieved by extracting the control module hardware out from the vehicle vessel and by reducing the on-board sensors. The control of the vehicle is conducted by a base station wirelessly telecommunicating with the vehicle. Furthermore, the reduction of the sensors also reduces the cost of the vehicle. For the purpose, in the vehicle, a single sensor featuring a CCD camera is mounted at the front of the vehicle. The images taken by this CCD camera are used both for obstacle avoidance and for underwater object searching. Experimental results show that the developed vehicle can successfully avoid the frontal obstacle while the operator in the base station can see the frontal view of the vehicle simultaneously. However, relative parameters of the image processing algorithm must vary for different time of the day and different whether. This implies that an adaptive image processing algorithm is necessary for operation in different situations.
\end{abstract}

\section{INTRODUCTION}

Autonomous Underwater Vehicles (AUVs) have gained people's interests due to their flexibility in underwater operation. In the past few years, a number of commercial AUVs have been developed for various practical applications, such as the investigation of underwater oilfields, the monitoring of near shore temperatures, the study of seabed profiles, the search for underwater resources, and the collection of scientific data $[1,5,7,8,12,13,15,16,19]$. However, the smallest vehicle among them is 1.5 meter in length and has problem accessing confined locations such as small caves, narrow channels, etc. Therefore, a Mini Autonomous Underwater Vehicles (MAUVs) capable of ex-

Paper Submitted 05/16/05, Accepted 08/31/05. Author for Correspondence: Chiu-FengLin. E-mail: chiufeng@mail.npust.edu.tw.

*Professor, Department of Vehicle Engineering, National Pingtung University of Science and Technology, 1, Hseuh Fu Road, Neipu, Pingtung, Taiwan. ploring these confined locations is desired. Furthermore, the current AUVs are expensive due to their sophisticate sensing and control system. This situation impedes the popularity of the AUVs. Thus, a cost effective AUV is preferred.

The reason that the sizes of the existing AUVs are large is that sophisticated sensing and control system is embedded on board, enough interior space is necessary for the hardware. Furthermore, sophisticated sensing system also keeps the cost of AUVs at a high level. Thus, to achieve mini size, this research proposes a suitable sensing and control architecture. This is accomplished by extracting the control module hardware out from the vehicle vessel and also by reducing the onboard sensors. In stead, the control is conducted by a base station which receives front view images from a CCD camera on the under water vehicle and then commands the motion of the vehicle thru wireless telecommunication.

In the past, several projects were devoted to the two key components of AUVs, namely sensing system and control module. The sensing system enables the AUVs to "see" the surrounding environment and to measure the required physical data. The sensors used on current underwater vehicles can be categorized as being either acoustic or non-acoustic. Acoustic sensors include side scan sonars, multi narrow beam sonars, and sub-bottom profilers $[5,7,13]$. On the other hand, nonacoustic sensors are typically image-based. Lasers and CCD cameras are generally used to evaluate the distance between the vehicle and an object and to identify the object's shape $[10,11,20]$. Usually, for the current AUVs, multi sensors system is implemented; each sensor is designated with a functional mission. Thus, to reduce the sensors, in this research, a single sensor system featuring a CCD camera is developed to fulfill the mission with multiple functionalities. The images from the CCD are used both for obstacle avoidance and for the operator to see the underwater scene thru wireless telecommunication. 
On the other hand, control module enables the vehicle to have autonomous capability. For this purpose, several different control architectures have been proposed. These control architectures can be categorized into hierarchical, behavioral, and hybrid architectures. Examples for hierarchical architecture are [6, 18]. Examples for behavioral architecture are [2, $3]$. Finally, examples for hybrid architecture are [9, 17]. The corresponding control modules of the above architectures are embedded in the vehicle vessel. In order to accommodate the control module hardware, enough space is required, leading to a substantial vehicle size. This problem is solved by extracting the control module out from the vehicle vessel as mentioned in the above section. A base station is developed to control the vehicle motion thru wireless telecommunication.

Finally, for most of the underwater vehicles mentioned previously, system dynamics is usually studied $[4,14]$. This is because they are designed to operate in situations with significant hydrodynamic forces. Sophisticated control algorithm must be developed to accommodate different situations. However, for this research, the vehicle is expected to operate in a still water environment like pond or a still deep sea. Also, the vehicle speed is relatively slow. Under these two conditions, the hydrodynamic forces won't be substantial. Thus, the system dynamics is ignored in this research.

\section{SYSTEM CONFIGURATION AND SPECIFICATION}

Figure 1 illustrates the basic configuration of the developed MAUV. It can be seen that the vehicle is equipped with four motors. The left and right motors enable the vehicle to move in the forward direction and to turn, while the front and rear motors enable it to dive or to surface. A needle type CCD camera is located at the front of the vehicle which enables it to "see" what lies in its path. Figure 2 presents a photograph of the MAUV and shows the use of a Nikko Sub168 module as part of the body. The use of Nikko Sub168 module is to attain the goal of cost effective by using the existing module on the market rather than self designing every components of the vehicle. In the developed underwater vehicle, only the middle section is from Nikko Sub168, which contains left and right motors and a wireless telecommunication receiver to receive commands from the base station. The rest of the underwater vehicle is developed by the current research project. This includes system configuration arrangement, front and rear sections design to provide extra room for CCD camera and another two motors, base station development for vehicle control and human interface, and wire- less telecommunication system interfacing between the vehicle and the base station.

Figure 3 illustrates the basic operation of the MAUV. As shown, the antenna is brought to the water surface by means of a buoyancy device to ensure that a clear signal is acquired. Table 1 provides a complete specification of the vehicle, while Figure 4 illustrates the configuration of the system hardware. The image acquired by the CCD camera is transmitted wirelessly to the base station. The transmitted data is captured by an image acquisition card and processed automatically to determine the position and nature of any obstacle in the vehicle's path. A motion controller then determines the necessary motor actuations required for the vehicle to avoid the obstacle. The corresponding actuation commands are transmitted wirelessly to the vehicle, which

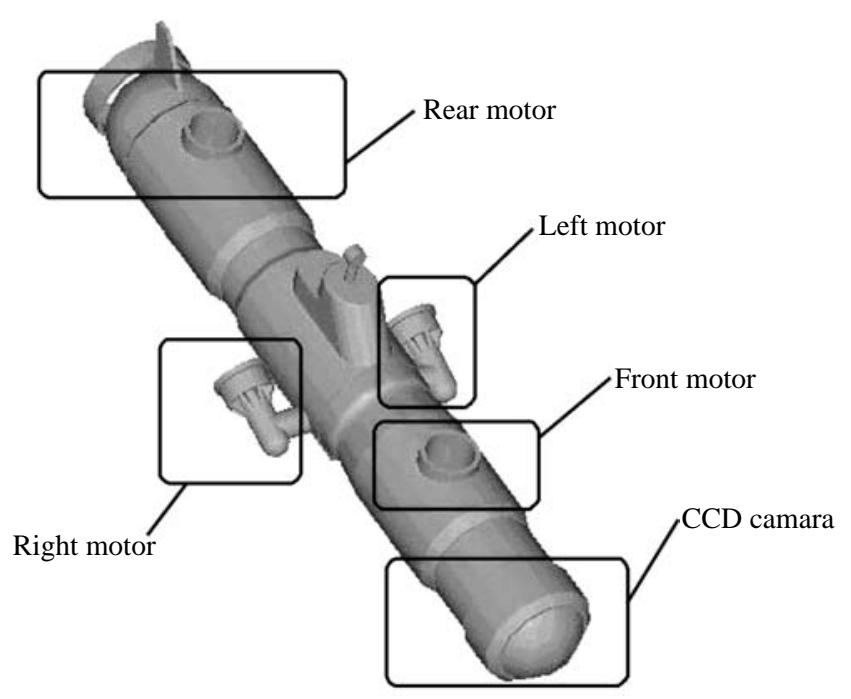

Fig. 1. Vehicle configuration.

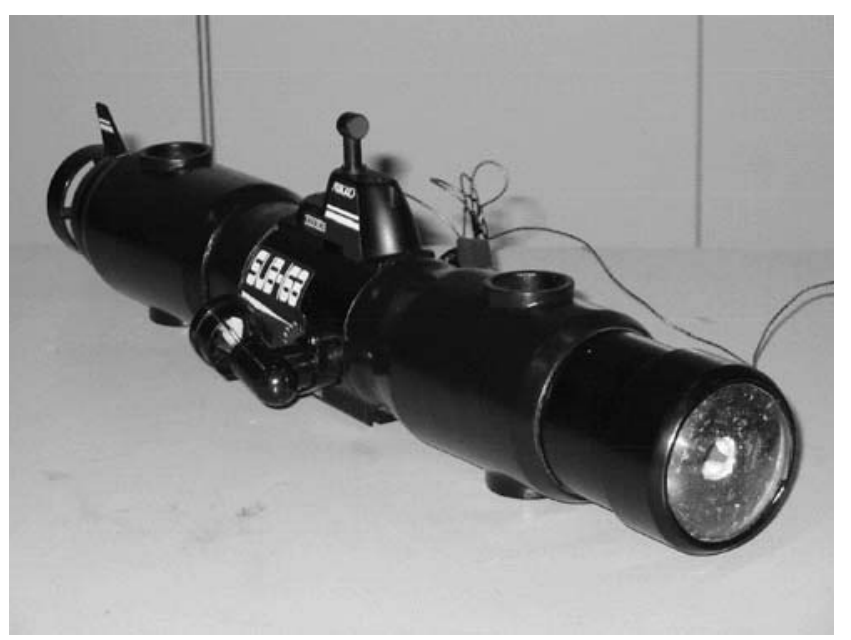

Fig. 2. Mini Underwater Autonomous Vehicle (MUAV). 


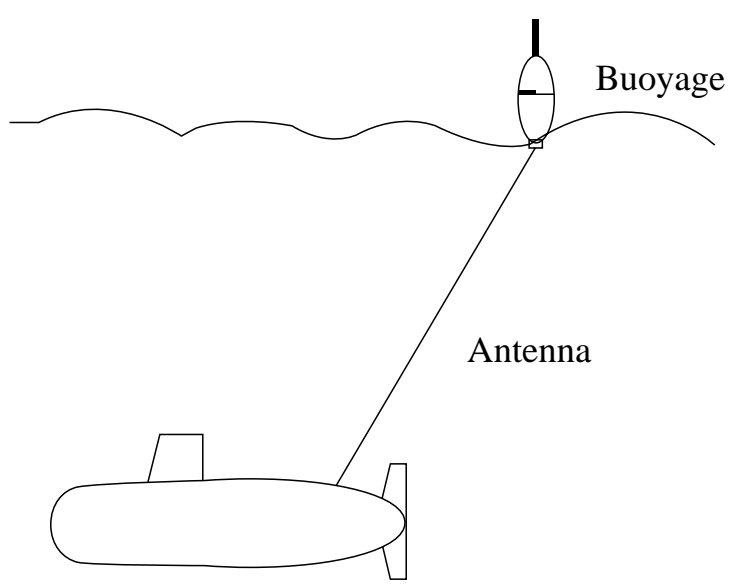

Under water vehicle

Fig. 3. Operational deployment of antenna.

Table 1. Vehicle specification

\begin{tabular}{lc}
\hline Size & $45 \mathrm{~cm} \times 12 \mathrm{~cm} \times 9 \mathrm{~cm}$ \\
\hline Weight & $1.75 \mathrm{~kg}$ \\
Operation depth & $1 \mathrm{~m}$ \\
Maximum speed & $0.15 \mathrm{~m} / \mathrm{s}$ \\
Operation time period & $90 \mathrm{~min}$ \\
Battery type & $\mathrm{Ni}-\mathrm{H} \times$ \\
Motor & $3 \mathrm{~V} / 3 \mathrm{~W} \mathrm{DC} \mathrm{motor} \times 4$ \\
Wireless transmission & $35 \mathrm{MHz} / 40 \mathrm{MHz}$ \\
Wireless transmission range & $15 \mathrm{~m}$ \\
Camera & $\mathrm{CCD} \mathrm{camera}$ \\
Front light & $1.2 \mathrm{~W} \mathrm{bulb} \times 2$ \\
Cornering radius & $0.25 \mathrm{~m}$ \\
\hline
\end{tabular}

activates its motors accordingly, thereby maneuvering around the obstacle. During this process, the acquired images are displayed on a monitor in the base station in real time to enable an operator to share the vehicle's view of the underwater scene.

\section{IMAGE PROCESSING AND OBSTACLE AVOIDANCE}

The core of the developed system is its image processing and obstacle avoidance unit. The basic steps of the image processing procedure are illustrated in Figure 5. Initially, the image captured by the CCD camera is acquired by an image DAQ card. Having acquired the image data, a grey level is assigned to each pixel in accordance with its luminance intensity. The grey level ranges from 0 to 255 , where a higher level corresponds to a brighter luminance. After the assign-

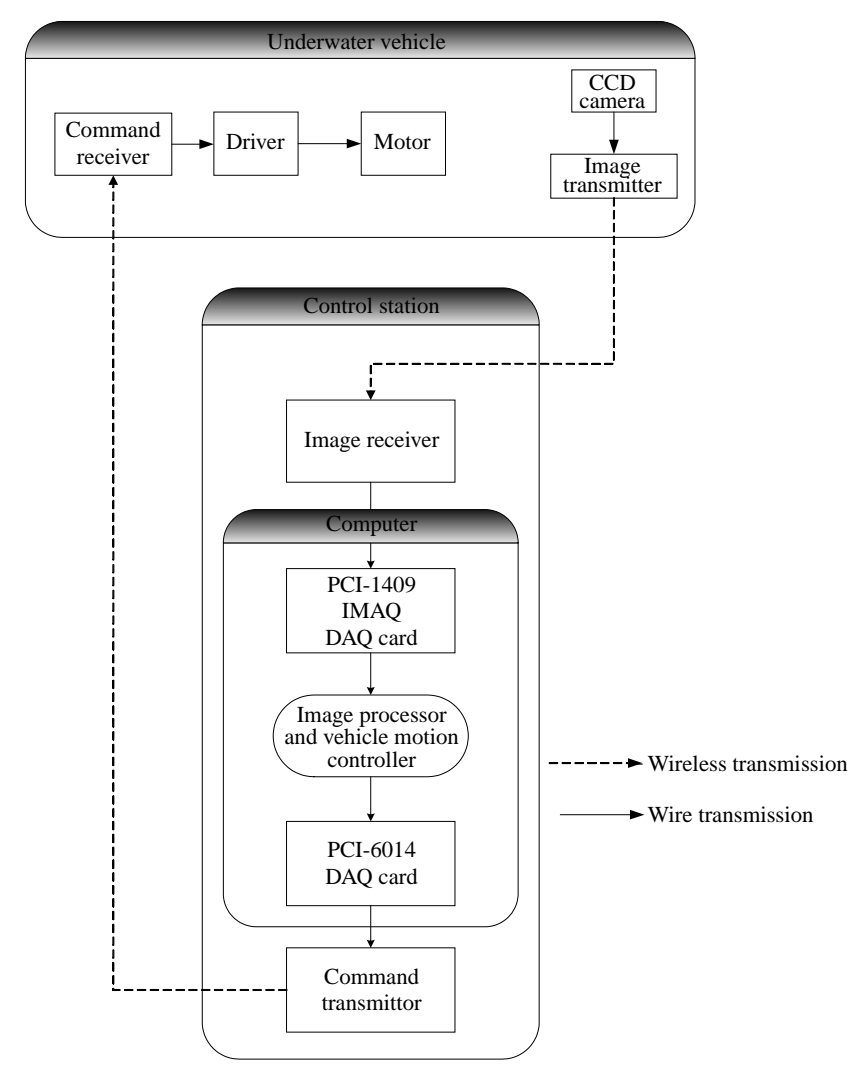

Fig. 4. System hardware configuration.

ing process, a sliding process is conducted in recognition of the fact that different operating environments may have different luminance intensities. By incorporating this process into the image processing procedure, the vehicle is provided with the ability to operate in a variety of weather conditions (e.g. sunny or cloudy) and at various times of the day (day time or night time). In the sliding process, the average grey level of each image frame is moved toward a desired value assigned according to different weather conditions. Suppose that the grey level of any pixel is given by $G(x, y)$ where $(x, y)$ corresponds to the position of the pixel in a frame. The average grey level of a frame is then calculated as:

$$
G_{\text {avg }}=\frac{1}{m n} \sum_{x=1}^{n} \sum_{y=1}^{m} G(x, y)
$$

where $n, m$ correspond to the range of the frame in horizontal and vertical axes. Furthermore, suppose that the desired average grey level is $G_{d e s}$. Sliding is then conducted by applying the following:

$$
G_{s}(x, y)=G(x, y)-\left(G_{a v g}-G_{d e s}\right)
$$

where $G_{s}(x, y)$ is the grey level value after sliding process. 


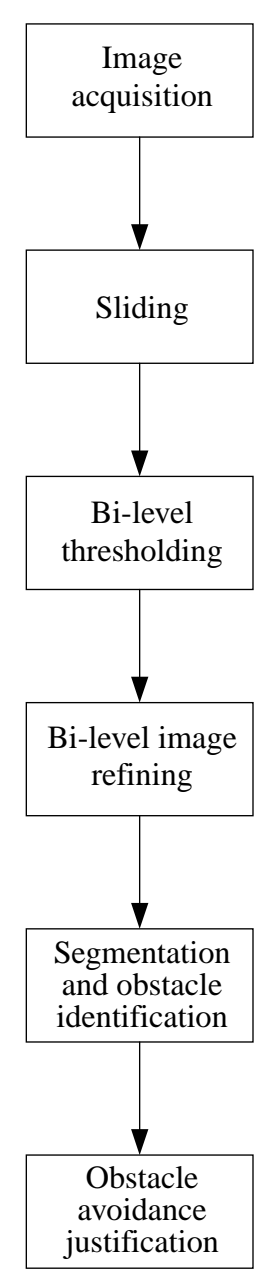

Fig. 5. Image processing procedure.

Following the sliding process, a bi-level thresholding process is performed in order to isolate the obstacle from the background. In this step, a threshold value is defined, denoted as $G_{t h}$. A pixel with a grey level value below this threshold is defined as belonging to the background, and the corresponding bi-level value is set to "0". Conversely, a pixel having a grey level above the threshold is defined as belonging to the obstacle, and the corresponding bi-level value is set to " 1 ". The rationale behind this bi-level thresholding process is that an obstacle reflects light to the CCD camera, whereas the background absorbs light. Therefore, pixels corresponding to the obstacle should have a higher grey level. Supposing that $G_{t h}$ is the bilevel value of each pixel, then the following expression can be applied:

$$
\text { If } G_{s}(x, y) \geq G_{t h} \text {, then } B(x, y)=1 \text {, else } B(x, y)=0
$$

This rationality also reflects a fact that if the obstacle is too far away from the CCD, it is not considered as an obstacle in the content of the current reasoning algorithm.

After the bi-level thresholding process, a bi-level image refining operation is conducted in which "salt and pepper" in the bi-level image is eliminated. In this operation, the term "salt" refers to individual pixels with a value of 1 located in a region of pixels with a value of 0 , while "pepper" refers to pixels with a value of 0 in a region of pixels with a value of 1 . The refining operation is performed using the following justification. Suppose a justification index, $C P$, is defined as

$$
C P=\sum_{i=1}^{1} \sum_{j=1}^{1} B(x+i, y+j)-B(x, y)
$$

The refining process is conducted by the following algorithm.

$$
\begin{aligned}
& \text { If } C P>4, \text { then } B(x, y)=1 \\
& \text { If } C P<4, \text { then } B(x, y)=0 \\
& \text { If } C P=4 \text {, then } B(x, y)=B(x, y)
\end{aligned}
$$

Once the bi-level image has been refined, it is segmented in order to identify the position of the obstacle relative to the vehicle. As shown in Figure 6, the image is divided into nine segments, corresponding to the top-left, top, top-right, left, middle, right, bottomleft, bottom, and bottom-right regions of the vehicle's front view. The total bi-level value of each segment is then calculated in order to test for the presence of an obstacle in the vehicle's forward path. The calculation procedure is performed as follows:

$$
\sum_{x=x_{\text {low }}}^{x_{\text {up }}} \sum_{y=y_{\text {low }}}^{y_{\text {up }}} B(x, y) \geq B_{\text {th }}
$$

where $B_{t h}$ is the obstacle threshold, $x_{\text {low }}$ and $x_{u p}$ are the lower bound and upper bound of a segment in the horizontal direction, and $y_{\text {low }}$ and $y_{u p}$ are the lower bound and upper bound of a segment in the vertical

\begin{tabular}{|c|c|c|}
\hline Top-left & Top & Top-right \\
\hline Left & Middle & Right \\
\hline Bottom-left & Bottom & Bottom-right \\
\hline
\end{tabular}

Fig. 6. Image segmentation. 
direction. If the condition in Eq. (5) is satisfied for a segment, then that segment contains a significant number of $B(x, y)=1$ pixels. In this event, the segment is assigned a bi-level value of 1 and is denoted as $S_{m, n}(t)$ $=1$.

Finally, a windowing process is conducted to confirm the existence of an obstacle in the segment. This is achieved by means of the following:

$$
\text { If } \frac{1}{n} \sum_{i=0}^{n-1} S_{m, n}(t-i \Delta t) \geq S_{t h} \text {, then } Q_{m, n}(t)=1
$$

where $S_{t h}$ is a threshold for the windowing process and a result of $O_{m, n}(t)=1$ indicates that an obstacle exists in the corresponding segment.

The above description reveals that the identification of an obstacle relates to several parameters including the bi-level threshold value, the desired average grey level of each image frame, the obstacle threshold, and the threshold for windowing process to ensure the existence of frontal obstacle. These parameter values are related to different times of day, different weather conditions, and mostly importantly the designated distance to "see" the frontal obstacle. The distance to "see" the obstacle relates to the desired distance from the obstacle the avoidance scheme is expected to takes action. Thus, one must first decide the distance to "see" the frontal obstacle and the parameters are assigned for different times of day and different weather conditions accordingly. The desired is that the vehicle can "see" the obstacle every time the vehicle reaches the designated distance. The relative performance can be evaluated by the recognition rate. The recognition rate is obtained by acquiring the percentage of obstacle recognition at every distance. In other words, for a distance, suppose $n$ image frames are acquired. By applying the above algorithm, $m$ frame are successful of obstacle recognitions. Then the recognition rate is $\frac{m}{n} \times 100$. One can expect that if $d_{\text {reg }}$ is the designated distance to "see" the frontal obstacle. The recognition rate should be $100 \%$ when the vehicle is at $d_{r e g}$ from the obstacle or closer than $d_{\text {reg }}$. On the other hand, if the vehicle is located at a distance larger than $d_{\text {reg }}$, the recognition rate will be smaller than $100 \%$.

Once the position of an obstacle has been identified, the obstacle avoidance routine is executed. The obstacle avoidance algorithm is shown in Figure 7. In this algorithm, the priority is for the vehicle to move directly ahead. However, if an obstacle exists in the middle segment, the default is to drive the vehicle to the left. If the left segment is occupied, the vehicle is moved to the right. If the right segment is also occupied, the vehicle is instructed to dive. If an obstacle exists in the bottom segment, the vehicle is driven in the left-downward

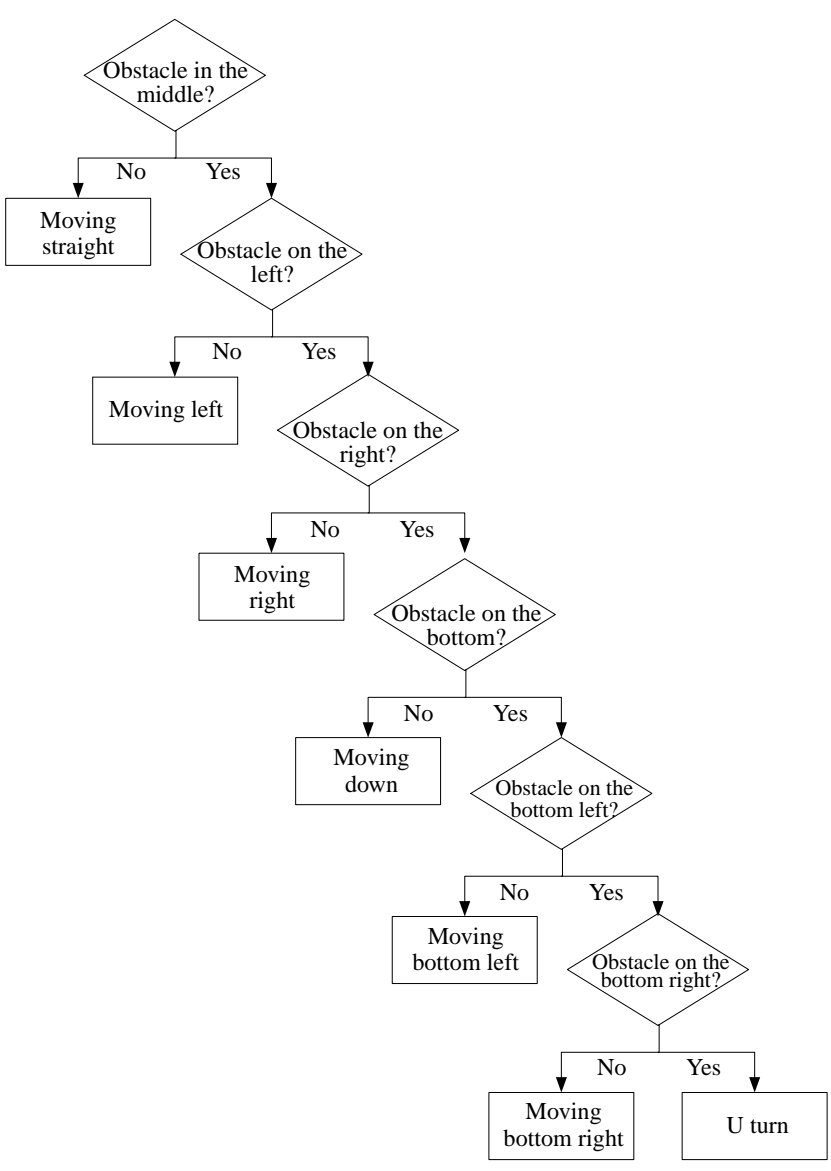

Fig. 7. Obstacle avoidance decision procedure.

direction. If this segment is also occupied, the vehicle moves in the right-downward direction. If this segment is occupied, the vehicle is unable to move in any forward direction and is instructed to execute a $U$ turn.

\section{EXPERIMENT RESULTS AND DISCUSSION}

This section describes the performance evaluation of the proposed MAUV. The evaluation tasks were conducted in an octagonal tank with overall dimensions of $270 \mathrm{~cm} \times 270 \mathrm{~cm}$. Experiments were performed under conditions representing different times of the day and the effect of using different image processing parameter settings was explored. The relative parameters for image processing were set such that the vehicle was capable of identifying an obstacle at a distance of $70 \mathrm{~cm}$.

Figure 8 shows the image processing results for two different obstacles positioned in front of the vehicle. From the images, it is clear that the obstacles have been successfully identified. However, it is noted that the identified shape does not match that of the obstacle precisely. A careful examination of the images shows 
that the bottom of the tank has been captured in both cases. This is because the surfaces of the tank also reflect a significant amount of light to the CCD camera and hence the grey levels of the corresponding pixels in the acquired image exceed the threshold value. In the same reasoning, the wall of the tank may also reflect significant light to the CCD camera. However, the walls of the tank can also be regarded as an obstacle in the vehicle's path and therefore it can be argued that the image processing results presented in Figure 8 are successful.

Figure 9 illustrates the dependency of the obstacle recognition rate on the distance of the vehicle from the obstacle. In the developed system, the distance to "see" the frontal obstacle is designated as $70 \mathrm{~cm}$. It can be seen that the recognition scheme functions correctly when the obstacle lies within a distance of $70 \mathrm{~cm}$. However, the recognition rate deteriorates at greater distances and the obstacle is essentially invisible to the vehicle at a range of approximately $90 \mathrm{~cm}$. When the obstacle lies within $70 \mathrm{~cm}$ of the vehicle, all of the reflected light is captured by the sensing system. However, at a greater distance, the reflected light is scattered over a wider area. Hence, less light is acquired by the CCD camera and the image processing performance is reduced. At a distance of $90 \mathrm{~cm}$, none of the reflected light reaches the CCD camera and therefore the obstacle cannot be detected. Of particular note is that the recognition rate at night time decreases from

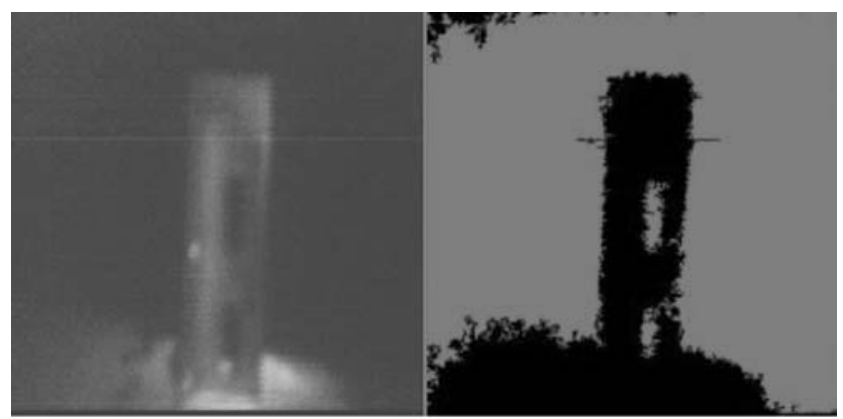

(a) Iron block (left: image from CCD camera, right: bi-level image)

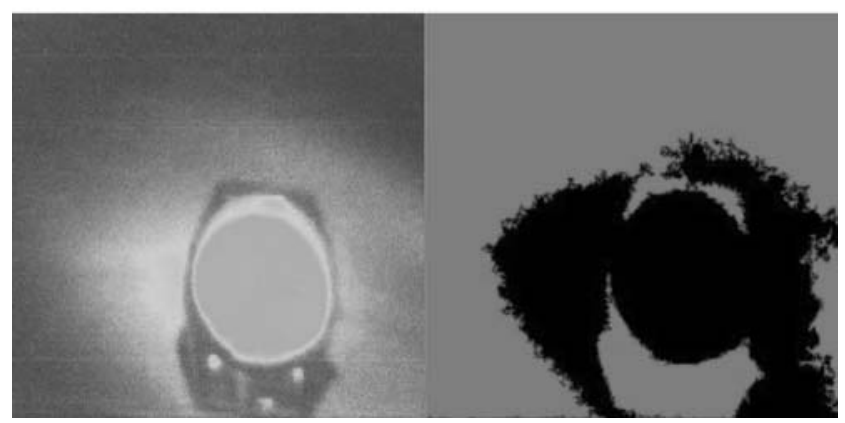

(b) Watch (left: image from CCD camera, right: bi-level image)
$100 \%$ at $78 \mathrm{~cm}$ to $0 \%$ at $83 \mathrm{~cm}$, i.e. the obstacle becomes invisible over a range of $5 \mathrm{~cm}$. However, under the other two experimental conditions, the obstacle becomes lost to the vehicle over a range of approximately $15 \mathrm{~cm}$. The reason for the discrepancy in these two ranges is that the light reflected from the obstacle is more easily scattered at night time and so the CCD camera will not capture extraneous light reflected from distant obstacles. The same situation applies to the background light which is essentially noises to the image processing. In other words, it can be argued that the reliability of the vehicle's obstacle detection capability is greater at night time since the level of environmental noise is reduced.

The image processing parameter settings are different in each of the three trials illustrated in Figure 9 since the luminance intensity varies in each case. In general, under sunny day time conditions, the $G_{d e s}$ value in the image processing sliding process is higher than under cloudy day time conditions because the intensity of the background light is greater on a sunny day. Therefore, a higher value of $G_{d e s}$ is required to eliminate the background noise. The $G_{d e s}$ value is also higher under sunny day time conditions than under any other operational conditions because the scattering of light

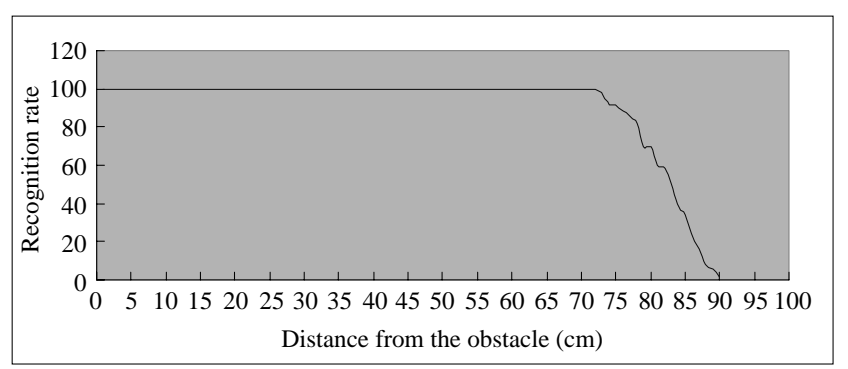

(a) Sunny day time result

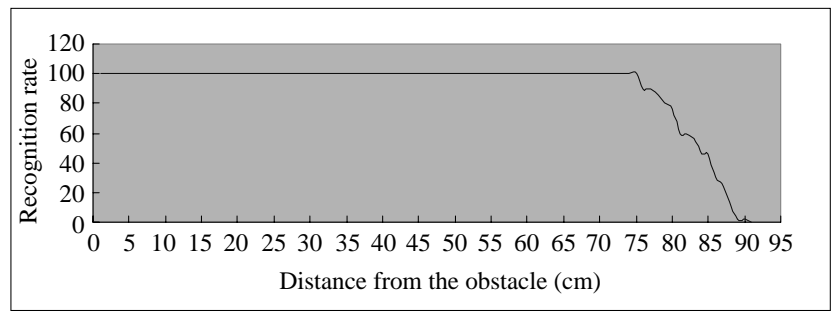

(b) Cloudy day time result

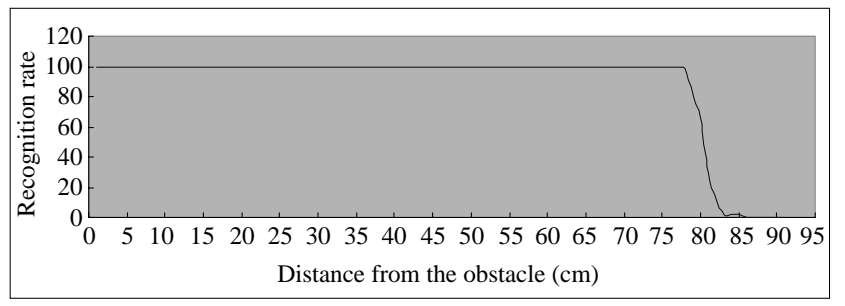

(c) Night time result

Fig. 9. Effect of obstacle distance on obstacle recognition rate.

Fig. 8. Results of obstacle recognition for different objects. 


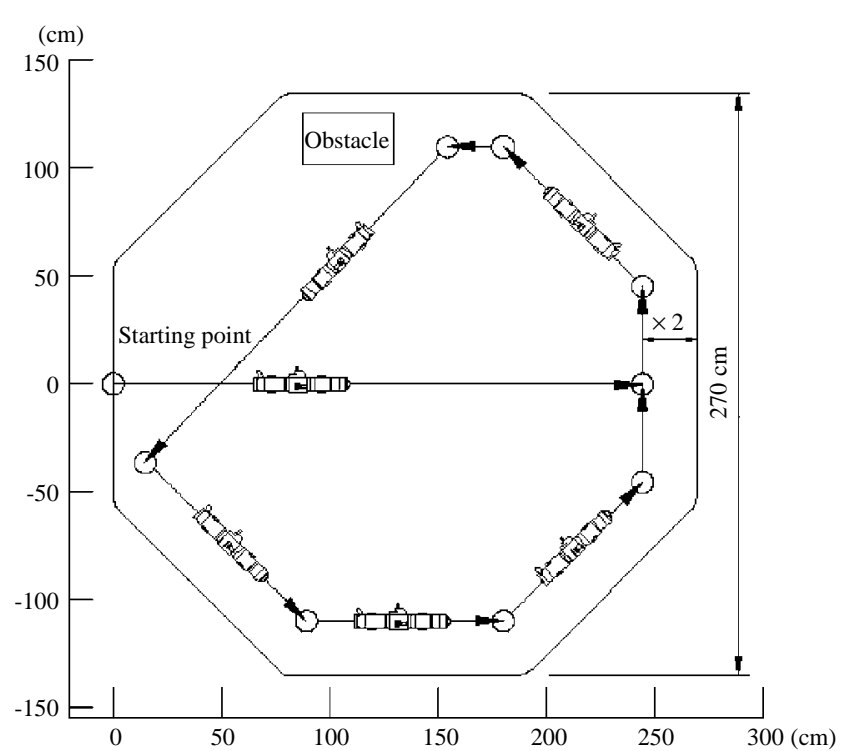

Fig. 10. Field test to evaluate obstacle avoidance capabilities.

from the background is strong in this situation. Accordingly, a higher threshold is necessary to prevent faulty detection. From the discussions above, it is clear that an adaptive obstacle identification algorithm is required if the system is to operate correctly under different lighting conditions.

Field tests were conducted to evaluate the obstacle avoidance capability of the developed MAUV. Figure 10 shows the path followed by the MAUV during a baseline field test. In this figure, the circles indicate the location of the vehicle center as it moved along the path. From its initial starting point, the vehicle proceeded toward the facing wall and then automatically turned left when the wall was detected. The notation "X2" indicates the turning distance from an obstacle. Note that a left turn was executed since, as described earlier, the algorithm defaults to a left turn when an obstacle is detected in the middle segment of the image. As shown in the figure, the vehicle successfully navigated around the boundary of the tank, turning to the left whenever a facing obstacle was detected. The results presented in this figure confirm the obstacle avoidance capability of the developed MAUV. The same field test was then repeated with different parameter settings. Figure 11 shows the results obtained for different settings of the $G_{t h}$ parameter. Note that the setting of 40 corresponds to the baseline field test described above. The results of Figure 11 reveal that a lower bi-level threshold value causes the vehicle to turn earlier. This is because at a lower value of $G_{t h}$, the area of $B(x, y)=1$ pixels struggles to attain the obstacle identification threshold, $B_{t h}$, required to trigger the setting of $O_{m, n}(t)=1$ at a far

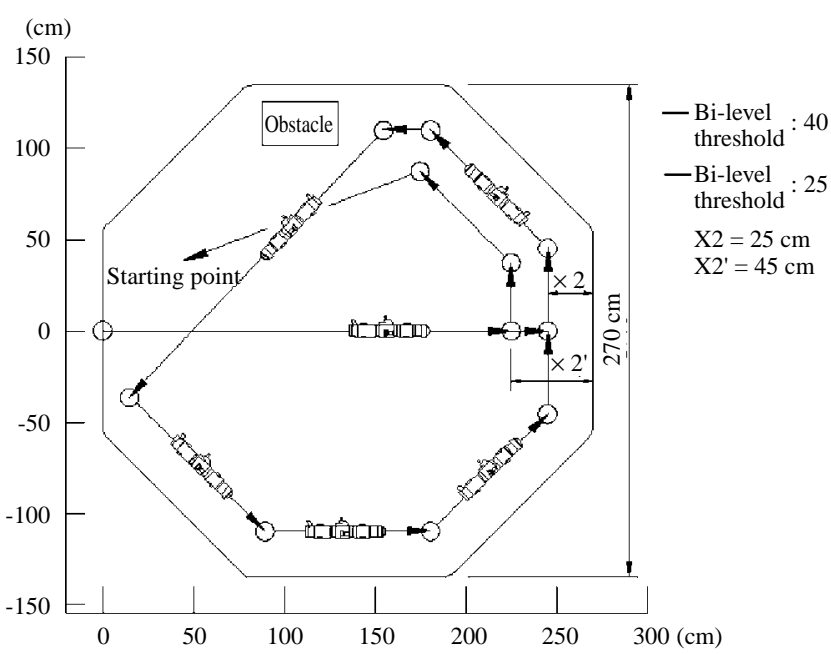

Fig. 11. Field test of obstacle avoidance with different bi-level thresholds.

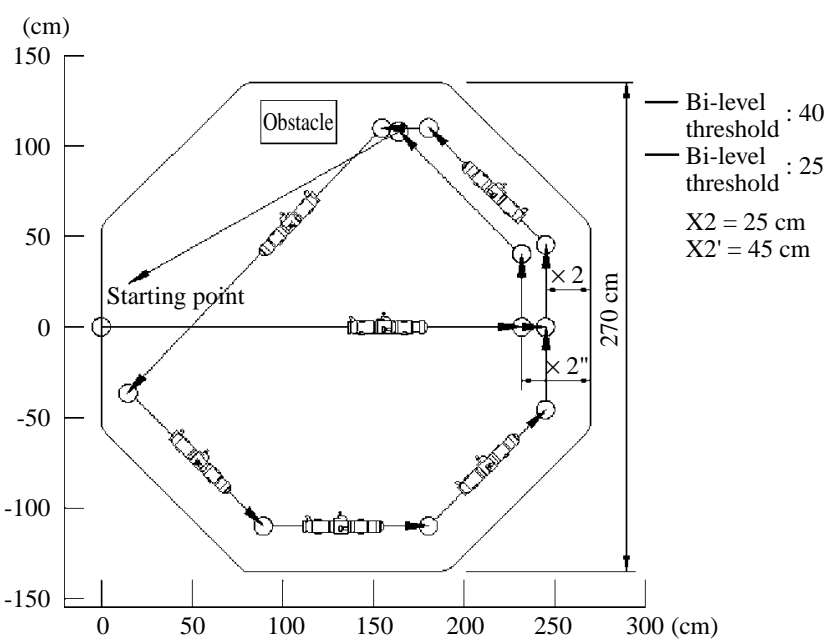

Fig. 12. Field test of obstacle avoidance with different obstacle thresholds.

distance. Figure 12 shows the results obtained with different obstacle threshold settings. The setting of 12,000 corresponds to the baseline field test. It is observed that a lower obstacle threshold causes the vehicle to turn earlier. This is because the pixels with $B(x, y)=1$ can satisfy a lower $B_{t h}$ value at a greater distance from the obstacle. Therefore, an obstacle is detected at a greater distance for a lower value of $B_{t h}$, causing the vehicle to turn sooner.

\section{CONCLUSION}

This study has successfully developed a mini autonomous underwater vehicle measuring $45 \mathrm{~cm}$ in length and having a cruising speed of $0.15 \mathrm{~m} / \mathrm{s}$. Using a frontmounted needle type CCD camera, the vehicle is able to 
detect and avoid obstacles in its forward path. The images acquired by the CCD camera are transmitted wirelessly to a controller located at a remote base station. The images are then processed automatically to identify the obstacle and appropriate command signals are generated to enable the vehicle to steer around the obstacle. The control commands are transmitted back to the vehicle via the wireless transmission link and the appropriate motors activated. The images taken by the CCD camera are also transmitted to a monitor in the base station such that an operator can share the forward view of the vehicle. The experimental results have shown that obstacles can be accurately identified provided that appropriate image processing parameters are specified. Therefore, provided that some form of adaptive algorithm is developed, the vehicle is capable of operating under different luminance conditions. Finally, the field test results have confirmed that the vehicle can successfully avoid obstacles placed in its path. It has been shown that different parameter settings, e.g. the bi-level threshold and the obstacle threshold, affect the distance at which the vehicle turns away from an obstacle.

\section{REFERENCES}

1. Anthony, J.H. and Marco, D.B., "Command, Control and Navigation," Special Issue on Autonomous Ocean Sampling Networks, Vol. 26, No. 4, pp. 466-477 (2001).

2. Arkin, R.C., Behaviour-Based Robotics, MIT Press, Cambridge, MA (1998).

3. Brooks, R.A., Cambrian Intelligence, the Early History of the New AI, MIT Press, Cambridge, MA (1999).

4. Evans, J. and Nahon, M., "Dynamics Modeling and Performance Evaluation of an Autonomous Underwater Vehicle," Ocean Engineering, Vol. 31, pp. 1835-1858 (2004).

5. Hagen, P.E. and Kristensen, J., "The HUGIN AUV "Plug and Play" Payload System," Proceedings of OCEANS 2002 MTS/IEEE, Biloxi, MS, pp. 156-161 (2002).

6. Hall, W.D. and Adams, M.B., "Autonomous Vehicle Taxonomy," Proceedings of the Symposium on Autonomous Underwater Vehicle Technology, Washington, DC, pp. 49-64 (1992).

7. Hill, A., "AUV Uptake in the Offshore Industry: Maintaining a Forward Momentum," Proceedings of UUVS 2002, Southampton, UK (2002)

8. Iwakami, H., Ura, T., Asakawa, K., Hujii, T., Nose, Y., Kojima, J., Shirasaki, Y., Asia, T., Uchida, S., Higashi, N., and Hukuchi, T., "Approaching Whales by Autonomous Underwater Vehicle," Marine Technology Society
Journal, Vol. 36, No. 1, pp. 80-87 (2002).

9. Kim, T.W. and Yuh, J., "Development of a Real-Time Control Architecture for a Semi-Autonomous Underwater Vehicle for Intervention Missions," Control Engineering Practice, Vol. 12, pp. 1521-1530 (2004).

10. Kondo, H., Ura, T., and Nose, Y., "Development of an Autonomous Underwater Vehicle "Tri-Dog 1" Toward Practical Use in Shallow Water," Journal of Robotics and Mechatronics, Vol. 13, No. 2, pp. 205-211 (2001).

11. Kondo, H. and Ura, T., "Navigation of an AUV for Investigation of Underwater Structures," Control Engineering Practice, Vol. 12, pp. 1551-1559 (2004).

12. Miller, D.P ., "Design of a Small, Cheap UUV for UnderShip Inspection and Salvage," Proceedings of the 1996 IEEE Symposium on Autonomous Underwater Technology, Monterey, CA, pp. 18-20 (1996).

13. Nicholson, P., "Review of De Beers Marine's M600 AUV Survey Operations for 2001/2002 Including Presentation of Acquired Data," Proceedings of UUVS 2002, Southampton, UK (2002).

14. Prestero, T., "Development of a Six-Degree of Freedom Simulation Model for the REMUS Autonomous Underwater Vehicle," Oceans 2001, Vol. 1, pp. 450-455 (2001).

15. Ura, T., Obara, T., Takagawa, S., and Gamo, T., "Exploration of Teisi Knoll by Autonomous Underwater Vehicle "R-One Robot," Proceedings of OCEANS 2001 MTS/IEEE, Honolulu, HI, pp. 456-461 (2001).

16. Ura, T., Kumagai, M., Sakskibara, T., Kimura, Y., Okumura, T., Shibasawa, K., Sasaki, M., and Matsushima, M., "Construction and Operation of Four Autonomous Underwater Vehicles for Lake Survey," Proceedings of UT02 IEEE, Tokyo, pp. 24-29 (2002).

17. Valavanis, K.P., Gracanin, D., Matijasevic, M., Kolluru, R., and Demetriou, G.A., "Control Architecture for Autonomous Underwater Vehicle," IEEE Control Systems Magazine, Vol. 17, No. 6, pp. 48-64 (1997).

18. Wang, H.H., Marks, R.L., and Rock, S.M., "Task-Based Control Architecture for an Untethered, Unmanned Submersible," Proceedings of the $8^{\text {th }}$ International Symposium on Unmanned Untethered Submersible Technology, Durham, NH, USA, pp. 1-12 (1993).

19. Yoerger, D.R., Bradley, A.M., Walden, B.B., Cormier, M.-H., and Ryan, W.B.F., "Fine-Scale Seafloor Survey in Rugged Deep-Ocean Terrain with an Autonomous Robot," Proceedings of ICRA 2000 IEEE, San Francisco, CA, Vol. 2, pp. 1787-1792 (2000).

20. Yu, S. and Ura, T., "Development of New Distance Measurement System for Autonomous Underwater Vehicles," Proceedings of the $19^{\text {th }}$ Conference of RSJ, CD-ROM, IN34 (2001). (in Japanese) 